\title{
A Context Ontology for Service Provisioning and Consumption
}

\author{
Oscar Cabrera, Xavier Franch, Jordi Marco \\ Universitat Politècnica de Catalunya (UPC) \\ UPC-Campus Nord, c/Jordi Girona 1-3, 08034 Barcelona, Spain \\ \{ocabrera I franch\}@essi.upc.edu,jmarco@1si.upc.edu
}

\begin{abstract}
Nowadays services as those provided by smart cities, health smart services, as well as common services (e.g., telephonic services, e-mail services), have a great economic impact for organisations and represent an important mean to deliver value to their consumers. The malfunctions of both the services themselves as well as the entities responsible for their execution and consumption might cause economic losses, consumers' dissatisfaction and even shorten the service life cycle, among other risks. To avoid malfunctions beyond maintaining quality levels desired, it is important to take into account the widest possible context information that cause either positive or negative effects around services and entities involved in their provisioning and consumption. In this paper, we propose an upper ontology for service provisioning and consumption from a service-centric perspective. Specifically, we focus on software services, although we could argue for more generic applications. The contribution is the analysis, evaluation and reuse of existing proposals on context models to identify the strengths and weaknesses of its current status as well as to identify contexts not yet considered, and consolidate an integrated view of these proposals. The ultimate intention is to provide a well-defined and consolidated infrastructure of context information as a common body of knowledge, that could be instantiated on variety of use cases, for example, to be instantiated by monitors as context information useful to be monitored, or to be used as context information that allows knowing which contexts affect a service when a user consumes it, among others.
\end{abstract}

Keywords-service provisioning; service consumption; service context; context model; context ontology; context hierarchy, context taxonomy.

\section{INTRODUCTION}

The proliferation of available services over the last years has increased the number of consumers and simultaneously stepped up both desired requirements and context-aware capabilities, not only in services but also in those entities supporting them, including the consumer himself. According to [1], "to provide adequate service for the users, applications and services should be aware of their contexts and automatically adapt to their changing contexts-known as context-awareness". In fact, context is key in the development of new services that will impact social inclusion for the emerging information society [2]. In this regard, services are increasingly requiring being aware to their context.

Context is a broad concept and several definitions are provided in the academic literature. In this paper we apply the definition given in Dey [3][4], "Context is any information that can be used to characterize the situation of an entity. An entity is a person, place, or object that is considered relevant to the interaction between a user and an application, including the user and applications themselves". This context definition could be used in several ways, e.g., to understand the place in which a service will be executed, to delimit a conversation among entities, to understand the behaviour of entities, to identify risks in service provisioning, among others.

We consider context from a service-centric perspective in which not only services but also entities supporting them, including the consumer, should be aware of context responsible of either positive or negative effects before, during or after their interaction. This kind of context is still poorly addressed and used; therefore, providing a common body of knowledge in this context perspective can be relevant and useful in a variety of use cases (e.g., helping in service development process, service monitoring, service adaptation, service improvement, etc.). The amount of knowledge that could be represented as context information may be extensive and it depends heavily on the entities identified in a certain interaction process, i.e. given an entity and its corresponding role in a process is possible to identify the context related. In this regard, it must be possible to represent context information as time, temperature, location, situation, etc.

The effort in context representation seems to go in the way of providing a well-defined infrastructure. In this sense, proposals on contexts have emerged in order to define models to represent the context knowledge. Hence, context models including ontologies, taxonomies and hierarchies are the engineering artefacts that have been proposed to provide welldefined structures and definitions of contexts. However, as it happens in many other areas, it does not exist a single model agreed by the scientific community; instead several proposals have emerged for specific or general purposes. These proposals may diverge in several matters: addressed facets, size, structure, terminology, underlying principles, etc. Whereby, it may be argued that it is necessary an effort to carry out a state of the art of context models specifically for ontologies, taxonomies and hierarchies in the area of services, to unify and consolidate existing context knowledge through a systematic roadmap mainly focused on analyse and evaluate the acquired information.

The aim of this paper is to provide a consolidated body of context knowledge expressed in an upper ontology for service- 
centric perspective. Specifically, we focus on software services, although we could argue for a more generic application. The roadmap is to identify and relate existing context models for services proposed in the academic research conducting a state of the art. As a result of such research, we have been able to identify the strengths and weaknesses of its current status. In addition, an attempt to reuse knowledge and provide new one will be addressed by means of an ontology following the guidelines provided in the formal ontology development process described in [5][6][7]. The importance of this work lies mainly in two lines, providing an overview of existing context models and making available a unified and consolidated ontology easy to be instantiated independent of the acting entity, specifically in the process of service provisioning and consumption.

The remainder of the paper is structured as follows. In section II is presented an introductory background in service provisioning and consumption, defining context, advantages to use an ontology for context representation and the ontology development process. In section III, the ontology specification is described. In Section IV the knowledge acquisition is addressed by performing a state of the art, providing an analysis and assessment of existing context models. In section $\mathrm{V}$ the ontology conceptualization and resulting ontology are presented. In section VI the resulting ontology is validated. In Section VII a scenario instantiating the context ontology is described. Finally, in Section VIII, we present the conclusions and future work.

\section{BACKGROUND}

In this section we give a brief description of the main streamlines that influence our work such as service provisioning and consumption processes, overview of context, advantages to use an ontology for context representation and the methodology used to conduct the formal ontology development process.

\section{A. Service provisioning and consumption}

In today's world, there are different kinds of services created to facilitate the life of the human in their daily tasks, i.e., these services have been developed to solve different needs according to certain requirements of different human contexts. As a result, an enormous explosion in the offering of services occurred, in fact it can be observed that for a given need, lots of these can be found. According to [8] there is a growth in consumer services driven by various social and economic factors and technology. Among these we find: rising demand for social services, size and role of the public sector, complexity of work environments, etc. The key of services lies in the value offered as the following definition specifies:

" a service is a means of delivering value to customers by facilitating outcomes customers want to achieve without the ownership of specific costs and risks" [8].

According to [9] delivering value has become a central issue in the discussion of whether service as a perspective or logic can offer something new for customers. From this perspective the same authors consider at least two aspects of service as a logic, viz. a logic for consumption and a logic for service provision. These two aspects of service logic are, of course, intertwined with and dependent on each other. Furthermore, the provider logic has to be geared towards the customer logic. Thus, the value creation depends widely on both aspects, but not limited to them.

We consider that the value creation is a process performed by different entities, where each entity has important roles and responsibilities to keep the value delivery. In order to identify those entities, it is necessary to understand the role of services in more complex structures such as the service-oriented architecture (SOA) defined as follows:

"A paradigm for organizing and utilizing distributed capabilities that may be under the control of different ownership domains. It provides a uniform means to offer, discover, interact with, and use capabilities to produce desired effects consistent with measurable preconditions and expectations" [10].

In order to have an extended vision of entities and roles involved in service provisioning and consumption, we have employed the extended service-oriented architecture (ESOA) provided in [11] as shown in Fig. 1.

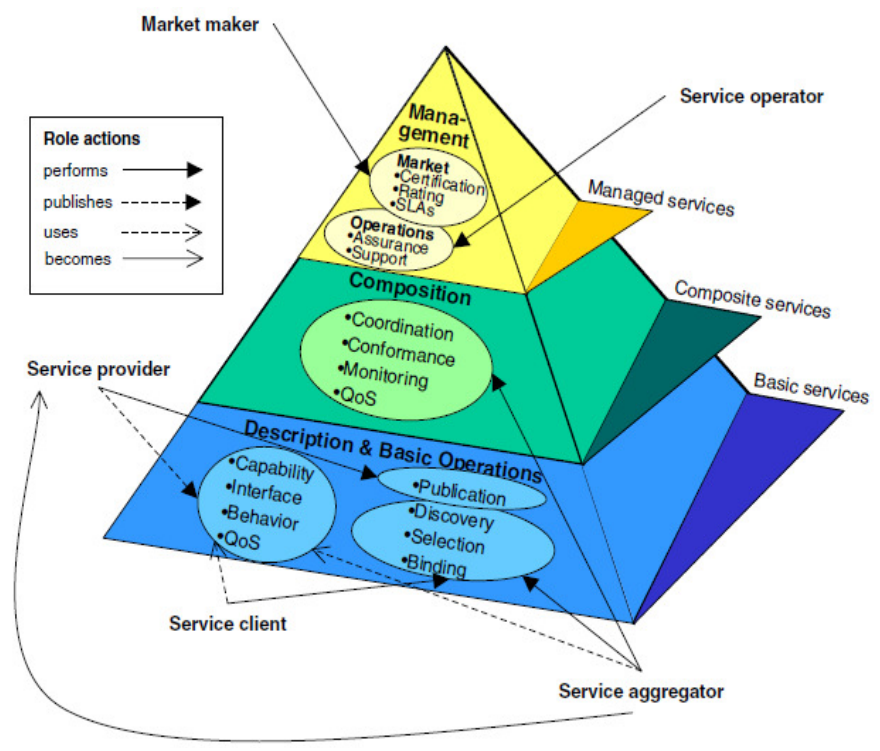

Fig. 1. Extended service-oriented architecture.

\section{B. Overview of context}

As already mentioned, the term context is widely used with very different meanings or very general definitions as the following, "context is the interrelated conditions in which something exists or occurs" [12]. This definition is too general and therefore, it does not help to understand what a context is and how it can be use, in order to apply the term effectively. In this sense we use the following context definition:

"Context is any information that can be used to characterize the situation of an entity. An entity is a person, place, or object that is considered relevant to the interaction between a user and an application, including the user and applications themselves" [3][4]. 
This context is very important, since it provides information about the present status of people, places, things and devices in the environment [13][14]. According to [15] the context information is useful to identify various situational circumstances, such as:

- The location and identity of the client who invoked the service, whether it is a person, or another service.

- The time at which the client invokes the service.

- The activity that the client is carrying out at the time it invokes the service.

- The preferences that the client may have defined prior to invoking the service.

- The security and privacy policies associated with the client of this service.

- The device (laptop, PDA, smartphone, etc.) that the client is using to invoke the service.

From a more technical point of view, the authors in [16] describe different forms of context that can be used by computers as: location, the presence of objects and people, temperature and blood pressure or more generally as any environmental factor that might influence the activities on a computer, provided it can be sensed.

The context information can be characterized by properties referred in literature as Quality of Context (QoC) indicators. It is defined in [17] as follows, "QoC is any information that describes the quality of information that is used as context information. Thus, QoC refers to information and not to the process nor the hardware component that possibly provide the information". This concept is a step forward once the context information has been identified, thus, it is out of the scope of this paper.

One of the major applications of context is in the area of context-awareness which refers to the ability to use context information. To avoid increasing complexity and allow the users to concentrate on his tasks, applications and services must be aware of their contexts and automatically adapt to their changing contexts-known as context-awareness [18]. One goal of context-aware systems is to acquire and utilize information in the context to provide services that are appropriate to particular people, place, time, event, etc. [1]. According to [19] a system is context-aware if it can extract, interpret and use context information and adapt its functionality to the current context of use.

In common services it is also important to take into account the context information. A good example is provided in [20] describing a restaurant service. In this case, it is argued that if context information is not provided, the restaurant would typically show the menu of the day. Instead, if context information is provided, the restaurant could eventually provide different suggestions depending on who is walking by. If parents with children walk by, the restaurant would show the children's menu; if a couple is looking at it in the evening, it would show the menu for a candle light dinner; and if it is hot and sunny in the afternoon, the restaurant would advertise the selection of ice cream.

\section{Advantages to use an ontology for context representation}

The representation of context information is usually carried out with the help of models that formalize collected context knowledge. In fact, as stated in [21] context is an information space that can be modelled as a directed state graph, where each node denotes a context, and edges denote the conditions for change in context. Each context is defined by a set of entities (typically including literal values, as well as real-world and information objects), a set of roles (for example, functions) that entities may satisfy, a set of relations between the entities, and a set of situations. Entities, roles, and relations are modelled as expressions over observables captured and inferred by the system at the appropriate level of abstraction.

In this regard, we use an ontology to represent context information. According to [22] an ontology is a formal explicit description of a domain, consisting of classes, which are the concepts found in the domain, also called entities. Representing context information based on an ontology is a challenge in the sense of describing in a precise and traceable manner this information. Ontologies seem to be the way to represent the knowledge concerning context in several domains. As stated by [23][24] ontologies are believed to be a key feature in the making of context-aware distributed systems due to the following reasons:

- Ontologies enable the sharing of knowledge by open dynamic agents (e.g. web services);

- Ontologies supply semantics for intelligent agents to reason about context information;

- Ontologies promote the interoperability among devices and computational agents.

According to [25] the reasons for developing context models based on ontology are the following:

- Knowledge Sharing. The use of context ontology enables computational entities such as agents and services in pervasive computing environments to have a common set of concepts about context while interacting with one another.

- Logic Inference. Based on ontology, context-aware computing can exploit various existing logic, reasoning mechanisms to deduce high-level, conceptual context from low-level, raw context, and to check and solve inconsistent context knowledge due to imperfect sensing.

- Knowledge Reuse. By reusing well-defined Web ontologies of different domains (e.g., temporal and spatial ontology), it is possible to compose large-scale context ontology without starting from scratch.

Other authors as in [26] mention other advantages of ontology for context modelling as the following:

- Amount of information to be delivered to the user can be reduced based on learner preferences and context ontology. 
- The user needs and expectations can be predicted to recommend suitable material based on the ontological inference mechanism.

- It enables formal analysis of domain knowledge for context reasoning from the explicitly defining context ontology.

- The main purpose of ontology-based context model is to enable semantic interoperability and to provide common understanding of the structure of context information among users.

\section{Ontology development process}

In the academic research there exist relevant methodologies on how to develop ontologies, some of these are the following: METHONTOLOGY [5][6][7], Uschold and King's [27], Grüninger and Fox's [28] and On-To-Knowledge [29], among others. In this work, the methodology used to develop the extended context ontology is based on the formal ontology development process described in METHONTOLOGY.

This methodology was selected because provides a set of activities and states well-structured and easy to follow; proposes the evolving prototype as the life cycle that better fits with the ontology life cycle; this evolving prototype allows to go back from any state to other if some definition is missed or wrong; activities like knowledge acquisition, documentation and evaluation are support activities that are carried out during the majority of the life cycle states; further, provides a set of deliverables to be produced after the execution of the activities using its attached techniques, thus documented ontology is ensured.

Activities and stages in METHONTOLOGY could be planed and arranged following an ontology life cycle in order to know when to perform the tasks to move from a given stage to the next and in how much depth. We rely on the life cycle selection based on the statements provided in [5] where the evolving prototype life cycle is used since the ontology grows depending on the needs. Indeed, this life cycle allows modifying, adding, and removing definitions in the ontology at any time. Thus, the activities and stages planned and arranged to be developed through the ontology life cycle are those related to specification, knowledge acquisition, integration, conceptualization and evaluation described as follows:

- Specification. This phase allows to provide domain, scope and purpose of the ontology starting with the following questions defined in [5][6]: what is the domain that the ontology will cover? Why the ontology is being built? What are its intended uses and endusers? Furthermore, to specify reasoning and scoping requirements of the ontology we define a set of competence questions following the guidelines provided in [28][30] in which is also mentioned that these questions are only a sketch and do not need to be exhaustive.

- Knowledge acquisition. Activity that provides the list of sources of knowledge and gives a rough description of how to carry out the knowledge acquisition. In this paper, this activity is carried out through a state of the art of existing context models.

- Conceptualization. Phase to conceptualize the acquired knowledge in a conceptual model that describes the problem and its solutions. This phase is useful to organize and convert an informally perceived view of a domain into a semi-formal specification using a set of intermediate representations that can be understood by domain experts and ontology developers [6][7]. The result of this phase is the conceptual model of the ontology.

- Integration. Reusing definitions already built into other ontologies instead of starting from scratch. As we have already mentioned, the essence of this work is to consolidate and integrate existing context knowledge providing a well-defined context infrastructure. Thus, the integration phase is carried out through the analysis, evaluation and selection of acquired context fulfilling the requirements defined into the specification phase.

- Evaluation. In order to avoid wrong definitions in the ontology an evaluation is needed. We carried out the evaluation during and until the end of the conceptualization phase to examine the ontology-based categorization to avoid possible errors [31].

The resulting formality of the ontology following the activities and stages previously defined reaches to a semiformal level corresponding to a conceptual view of the ontology [5] provided in this paper. The next sections report the stages and activities applied to our work.

\section{SPECIFICATION}

As we have mentioned, specification phase is needed to determine the domain, scope and purpose of the ontology. This information is an important base supporting the knowledge acquisition activity in the inclusion and exclusion of concepts coming from existing context models, mostly considered those fulfilling the specification. In the remainder of this section we answer the questions specified in the previous section, that according [5][6] this questions and their corresponding answers are useful to carry out this phase.

\section{A. Domain, purpose and scope}

The domain of the ontology is focusing on contexts causing either positive or negative effects on entities involved in service provisioning and consumption. In this sense, the scope of the ontology is delimited by relevant entities surrounding the domain specified. Following the bottom-up approach the central idea is that context is always connected to an entity, i.e., context information is produced by characterizing the situation of each entity participant in the interaction given in a process.

At this point of acquired knowledge based on both the information provided in Section II.A, and the brainstorming generated with service experts, we have identified basic entities surrounding the service provisioning and consumption. To show them we propose the following diagram depicted in Fig. 2. viewed as a service-centric perspective. 


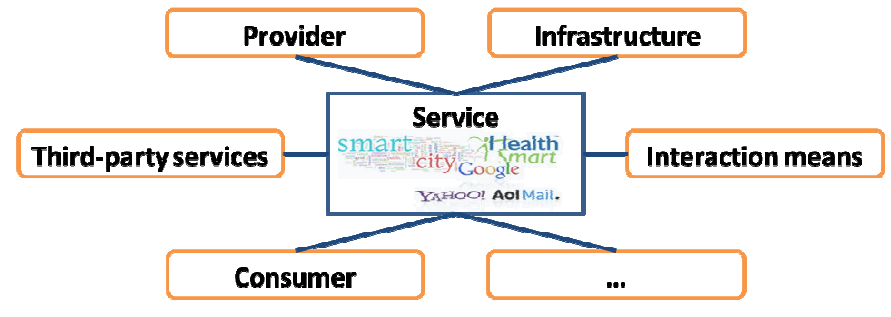

Fig. 2. Context viewed as a service-centric perspective.

It is worth noting, that this perspective is a basic view of entities that at least should exist in service provisioning and consumption, but it could be extended. In this regard, in the next section an effort to identify further entities fulfilling the specification requirements and that could be integrated in the service-centric perspective proposed is made. In addition, a compilation of context information affecting each of the entities identified is addressed.

The purpose of the ontology is to provide structured context knowledge from a service-centric perspective. According to academic literature, there is context information provided by conceptual models yielding context hierarchies for different domains, but despite the various attempts to develop a taxonomy for context information, there is no generic context categorization applied altogether in service-centric perspective. In this sense, our motivation lies in the lack of ontologies oriented to consolidate and provide an integrated view of context information in service provisioning and consumption.

Given the service-centric perspective of the ontology its use cases and end-users can be varied depending of the entity involved in the interaction with a service. For instance, the ontology can be used by consumers in order to know which kind of context information affects their interaction with a service (e.g., the interaction between a person and smart parking service, maybe it is affected by social aspects such as strikes), also the ontology can help consumers to understand their own context affecting the interaction with a service (e.g., language of the user, situation of the user, etc.), from a more technical use case, the ontology can provide context information useful for monitors (e.g., sensors, systems, etc.) to observe services and entities involved.

In order to support the scope of the ontology in the sense of delimit contexts integrating its structure we have established a series of competence questions following the guidelines provided in [28][30]. In this sense, we have oriented the competence questions mainly to identify both the entities involved in service provisioning and consumption and the context for each entity identified. According to [32] competency questions can be used for scoping and evaluation, where scoping entails knowing what must not be in the ontology. To define and establish competence questions in our domain the background provided in Section II and the proposed service-centric perspective depicted in Fig. 2. were taken as primary source of information.

Thus, the competence question oriented to identify and establishing entities in our domain carried out in the next section as part of the analysis and evaluation of existing context models is defined as follows:
- Given both the definition of context in Section II.B and the background of service provisioning and consumption in Section II.A. What are the entities involved in service provisioning and consumption that can be characterized as context? As baseline we have identified the entities depicted in Fig. 2. such as service, customer, service provider, service infrastructure, interaction means and third-party services.

Hence, those entities responding the previous competence question will be included as a concept in the proposed servicecentric perspective. To retrieve context information involved in each entity identified, we took as bases the entities already specified in Fig. 2. to formulate the following competence questions, for brevity's sake, we only define three of them:

- Given the statement that a service provider is the entity responsible of delivering a service. What are the contexts involved in a service provider affecting the service provisioning and consumption?

- Given the statement that a service needs an infrastructure to be delivered, used and provided with certain functionality. What are the contexts involved in the service infrastructure affecting the service provisioning and consumption?

- Given the statement that customers sometimes need means to interact with a service (e.g., smartphones, computer, another service, etc.). What are the contexts involved in an interaction mean affecting the service provisioning and consumption?

Hence, context information answering the competence question for each entity identified will be characterized in the ontology. For brevity's sake, answers to previous competence questions were omitted since the whole context is defined in the resulting ontology, depicted in Fig. 6. The aim of this ontology is the representation of context information (CI) affecting the behaviour of entities identified based on the service-centric perspective depicted in Fig. 2. To illustrate the aforementioned, we provide the diagram showed in Fig. 3. in which each entity has an abstract context composed by an aggregation of context information or context elements affecting one or more entities.

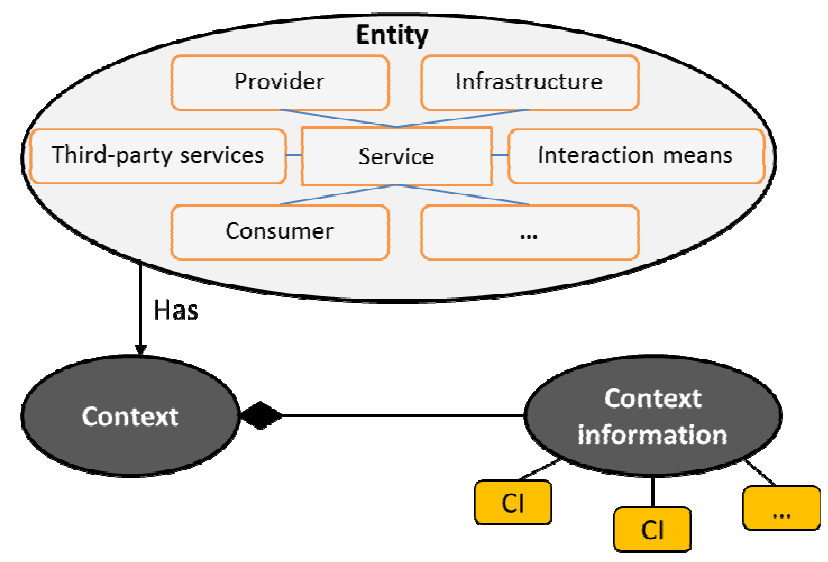

Fig. 3. Overview of the proposed work. 
Based on the previous information, we are prepared to decide about the inclusion or exclusion of concepts in the ontology as well as about their hierarchical structure. We summarize in TABLE I the information gathered in the specification phase using the ontology specification document provided in [5].

TABLE I. ONTOLOGY REQUIREMENT SPECIFICATION.

\begin{tabular}{l|l}
\hline \multicolumn{2}{|c|}{ Ontology requirement specification document } \\
\hline $\begin{array}{l}\text { Domain: } \\
\text { Purpose: }\end{array}$ & $\begin{array}{l}\text { Service provisioning and consumption } \\
\text { Providing structured context knowledge from a service-centric } \\
\text { perspective }\end{array}$ \\
$\begin{array}{l}\text { Level of } \\
\text { formality: }\end{array}$ & Semi-formal \\
$\begin{array}{l}\text { Scope: } \\
\text { Sources of } \\
\text { knowledge: }\end{array}$ & $\begin{array}{l}\text { Context information affecting entities involved in the domain } \\
\text { Background addressed in Section II, brainstorming and the } \\
\text { study carried out in the following section. }\end{array}$ \\
\hline
\end{tabular}

\section{KNOWLEDGE ACQUISITION}

This section is addressed performing a state of the art of existing proposals on context models, highlighting the analysis, evaluation and reuse of them to identify the strengths and weaknesses of its current status as well as to identify contexts not yet considered, and consolidate an integrated view of these proposals attending a common body of knowledge. According to [32] speed and reliability may be increased by using an existing ontology as the starting point and basis for guiding knowledge acquisition.

\section{A. State of the art}

Different proposals of context models have been addressed in the academic literature providing benefits mainly for specific domains. These proposals outline context models in form of ontologies, taxonomies or informal hierarchies. In many cases these models and definitions are weak and incomplete, yet they have led to serious discussions of the subject. Furthermore, context models despite the various attempts to develop a taxonomy for contexts, do not provide a profound and generic context categorization. Most of the introduced context ontologies focus on isolated context-aware applications.

Relevant context information differs from a domain to another and depends on the effective use of this information. Although most of the context information provided by existing models can be applied in the domain of service provisioning and consumption, other context information has been neglected. In fact, an upper ontology focused on providing context affecting the interaction among entities involved in the service-centric perspective has not been yet considered at all in context ontologies.

In the remainder of this section a brief review of research works on context models is presented, while an attempt is made to analyse and evaluate each context proposals. In order to carry out systematically the review, we have selected a set of relevant and objective general criteria provided in [33] to assess the context model proposals. In addition, we have included criteria that differ among the different works, such as domain, hierarchies, depth level, nodes, context definitions and development method. These criteria are specified as follows:
- Domain. Area or topic in which the context model was proposed. E.g., context-aware, smart environments, ubiquitous and pervasive computing, etc.

- Subject. Entity or subject for which the context model is described. E.g., user, service, mobiles, etc. We use flexible if the context model can be applied for different subjects or entities, otherwise, specific.

- Formalism. Type of formalism or model used to represent the context. E.g., ontology-based, taxonomybased, graph-based, etc. Whether a formalism is not used or at least it is not presented we use unemployed.

- Level. Level of formality of the proposed model. We use higher if the model was specified in a formal language, otherwise, lower.

- Hierarchies. Whether the model proposed clearly defines context hierarchies. We use defined if at least the first level hierarchy is presented, semi-defined if no formal categorization is defined, in this case the proposed context is considered as first level hierarchy, otherwise, undefined.

- Depth level. Maximum depth level of the model proposed, i.e., number of hierarchy levels defined in the context model. We consider levels of classes and subclasses, but instances of properties or values are discarded.

- Nodes. Number of nodes presented in the context model. We consider as nodes, classes and subclasses; instances of properties or values are discarded.

- Definitions. Whether each context provided in the model is defined. We use complete if all levels of contexts presented in the model are defined, first level if at least contexts of the first level hierarchy are defined, partially if at least a notion of the context addressed is presented, otherwise, null.

- Method. Type of method used to develop the context model, e.g., user-centric perspective, reusing context knowledge, etc.

The information presented in TABLE II was acquired based on previously defined criteria and represents both an overview of research works on context models and starting point to consolidate representative context knowledge as part of the paper roadmap. In fact, the purpose at this stage is to provide a general landscape of context model proposal whilst learning different issues such as, type of recurrent information, type of issues that can be observed during the data analysis, type of information that can be used for further analysis, among others.

Through the results provided in TABLE II we identified different issues in the context models addressed. Related to context models definitions we have observed that a set of these context models were derived from existing ontologies yielding distributed definitions, i.e., the context definitions mostly were omitted and delegated for reused ontologies. 
TABLE II. OVERVIEW OF CONTEXT MODELS PROPOSALS.

\begin{tabular}{|c|c|c|c|c|c|c|c|c|c|}
\hline Proposal & Domain & Subject & Formalism & Level & Hie rarchies & Depth level & Nodes & Context definitions & Development method \\
\hline W. Schilit et al. [34] & Context-aware computing & Specific & Unemployed & Lower & Semi-defined & 2 & 15 & Null & Unspecified \\
\hline G. Chen et al. [35] & Context-aware computing & Specific & Unemployed & Lower & Semi-defined & 2 & 20 & Partially & Unspecified \\
\hline A. Dey [3][4] & Ubiquitous computing & Specific & Unemployed & Lower & Semi-defined & 1 & 4 & Partially & Unspecified \\
\hline K. Mitchell [36] & Context-aware computing & Specific & Unemployed & Lower & Semi-defined & 2 & 27 & Partially & Unspecified \\
\hline A. Schmidt et al. [37] & Context-aware computing & Flexible & Model-based & Lower & Defined & 4 & 17 & Partially & A working model proposed \\
\hline X. Wang et al. [25] & $\begin{array}{c}\text { Pervasive computing } \\
\text { environments }\end{array}$ & Flexible & Ontology-based & Higher & Defined & 2 & 14 & Partially & Unspecified \\
\hline D. Preuveneers et al. [38] & $\begin{array}{c}\text { Smart environments and } \\
\text { movile computing }\end{array}$ & Specific & Ontology-based & Higher & Defined & 4 & 30 & First level + partially & Unspecified \\
\hline H. Chen et al. [24] & $\begin{array}{c}\text { Ubiquitous and Pervasive } \\
\text { Applications }\end{array}$ & Flexible & Ontology-based & Higher & Defined & 2 & 17 & Complete & $\begin{array}{l}\text { Use case scenarios to reuse } \\
\text { vocabulary }\end{array}$ \\
\hline H. Kranenburg et al. [39] & Context-aware computing & Specific & Unemployed & Lower & Semi-defined & 1 & 5 & Partially & User-centric perspective \\
\hline A. Zimmermann et al. [40] & Context-aware computing & Flexible & Categorization-base & Lower & Defined & 2 & 23 & Complete & $\begin{array}{c}\text { Based on available structuring } \\
\text { approaches }\end{array}$ \\
\hline B. Medjahed et al. [41] & Web service environments & Flexible & $\begin{array}{l}\text { Ontology-based } \\
\text { categorization }\end{array}$ & Higher & Defined & 4 & 26 & Partially & $\begin{array}{l}\text { Categorization from client and } \\
\text { provider point of view }\end{array}$ \\
\hline P. Eugster et al. [42] & Context-aware computing & Flexible & Unemployed & Lower & Semi-defined & 2 & 6 & First level + partially & Based on category scope \\
\hline K. Bandara et al. [43] & Web service environments & Specific & Ontology-based & Lower & Defined & 2 & 16 & Complete & $\begin{array}{c}\text { Empirical approach by looking } \\
\text { at three case studies }\end{array}$ \\
\hline T. Stavropoulos et al. [44] & Smart environments & Specific & Ontology-based & Higher & Defined & 1 & 3 & Partially & $\begin{array}{l}\text { Ontology Development } 101 \\
\text { Guide }\end{array}$ \\
\hline A. Ranganathan et al. [45] & $\begin{array}{c}\text { Pervasive computing } \\
\text { environments }\end{array}$ & Specific & Ontology-based & Higher & Defined & 2 & 25 & Null & Predicate logic \\
\hline M. Grassi et al. [46] & Smart environments & Flexible & Ontology-based & Higher & Defined & 5 & 77 & Partially & $\begin{array}{c}\text { Ontology framework + binding } \\
\text { ontologies approach }\end{array}$ \\
\hline C. Niederée et al. [47] & $\begin{array}{l}\text { Cross-system } \\
\text { personalization }\end{array}$ & Specific & Class model-based & Lower & Defined & 3 & 16 & Complete & $\begin{array}{c}\text { Based on Unified User } \\
\text { Context Modeling (UUCM) }\end{array}$ \\
\hline $\begin{array}{l}\text { M. Kaenampornpan et al. } \\
{[48]}\end{array}$ & Context-aware computing & Specific & $\begin{array}{c}\text { Triangular structure } \\
\text { based }\end{array}$ & Lower & Defined & 2 & 10 & First level & $\begin{array}{c}\text { Approach } \\
\text { based on Activity Theory }\end{array}$ \\
\hline M. Strimpakou et al. [49] & $\begin{array}{c}\text { Pervasive computing } \\
\text { environments }\end{array}$ & Flexible & Ontology-based & Higher & Defined & 2 & 19 & First level + partially & $\begin{array}{l}\text { Based on Semantic Context } \\
\text { Entity (SCE) identification }\end{array}$ \\
\hline C. Bolchini et al. [50] & Context-aware computing & Specific & Tree-based ontology & Higher & Defined & 1 & 7 & First level & Context Dimension Tree \\
\hline K. Henricksen et al. [51] & $\begin{array}{c}\text { Pervasive computing } \\
\text { environments }\end{array}$ & Specific & Graph-based & Lower & Defined & 2 & 10 & Partially & Object-based approach \\
\hline H. Yang et al. [52] & $\begin{array}{l}\text { U-Learning } \\
\text { Environments }\end{array}$ & Specific & Ontology-based & Higher & Defined & 3 & 27 & Partially & Unspecified \\
\hline M. Blake et al. [53] & Service-oriented computing & Specific & Graph-based & Lower & Defined & 1 & 3 & First level & Unspecified \\
\hline M. Golemati et al. [54] & User context and profiling & Specific & Ontology-based & Higher & Defined & 2 & 17 & First level & Top-down approach \\
\hline H. Shen et al. [55] & Mobile Web services & Specific & Ontology-based & Higher & Defined & 3 & 28 & First level + partially & Unspecified \\
\hline K. Pathan et al. [56] & Context-aware computing & Flexible & Ontology-based & Lower & Defined & 2 & 10 & First level & Based on W4H design space \\
\hline S. Scerri et al. [57] & $\begin{array}{c}\text { Pervasive computing } \\
\text { environments }\end{array}$ & Specific & Ontology-based & Higher & Defined & 2 & 36 & First level & $\begin{array}{l}\text { "Shared conceptualisation" } \\
\text { standards }\end{array}$ \\
\hline J. Kim et al. [58] & U-healthcare environments & Flexible & Ontology-based & Higher & Defined & 4 & 39 & Partially & Context modeling combination \\
\hline K. Sudhana et al. [26] & E-learning environments & Specific & Ontology-based & Higher & Defined & 3 & 24 & First level + partially & $\begin{array}{c}\text { Information acquisition } \\
\text { perspective }\end{array}$ \\
\hline A. Dix et al. [59] & Context-aware computing & Specific & Taxonomy-based & Lower & Defined & 1 & 4 & First level + partially & Unspecified \\
\hline
\end{tabular}

One of the most referenced ontologies that reuse other ontologies is provided by $\mathrm{H}$. Chen et al. in [24] which present an ontology called SOUPA in which part of the vocabulary addressed is adopted from a number of different consensus ontologies including [60][61][62][63][64][65][66].

Another important issue is related to the domain and hierarchies, although different domains were analysed, a common core of context surrounding them can be observed. However, different context hierarchies are provided which breaks defining a context baseline. The main problem that we have observed related to hierarchies is the level of abstraction that proposals try to transmit in the model, sometimes is very specific or very generic. In this sense, a further analysis and evaluation of hierarchies is addressed in following sections. Moreover, information related to depth level, nodes and context definition is used for further analysis and assessment on specific characteristics presented in the context models proposed such as size, definition completeness, among others. It is widely described in the remainder of this section.

\section{B. Context models caracteristics}

This section is addressed analysing and evaluating the structural characteristics of the proposed context models in terms of its size (quantity) and definition coverage (quality).

- Size: we evaluated the size considering the amount of nodes (representing context information) specified in the context models and its level of depth. Through this criterion, the most extensive context models are proposed by M. Grassi et al. [46] with 77 nodes, followed by J. Kim et al. [58] with 39, and S. Scerri et al. with 36 nodes. In the first case, the presented context model has a 5-level depth, second case a 4-level depth and the third case a 2-level depth. In this regard, the correlation presented in context models between the number of nodes and the depth level is depicted in Fig. 4. 


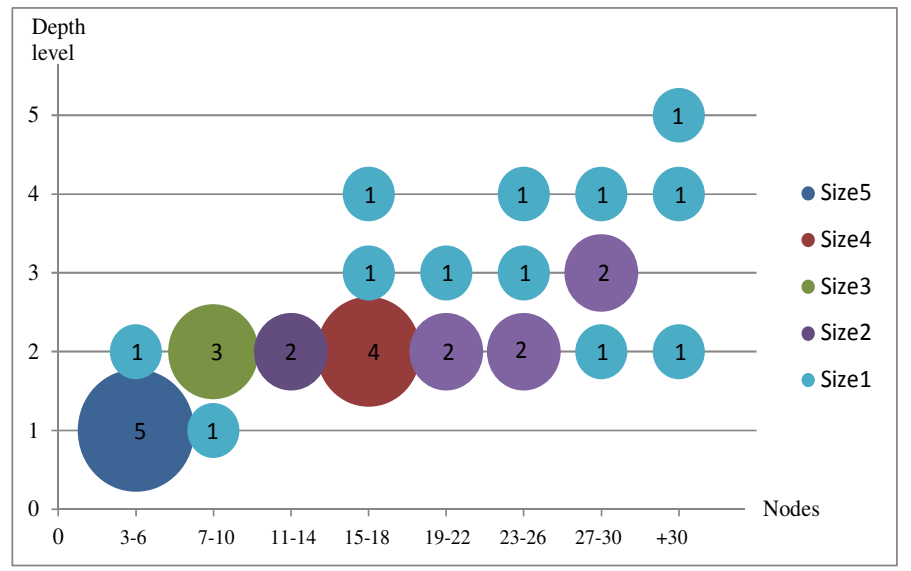

Fig. 4. Correlation map between nodes and depth levels.

We can observe that context models presenting nodes between 3 and 6 were usually developed in 1-level depth (5 proposals). In the mid-range area, context models between 15 and 18 nodes were usually developed in a 2-level depth (4 proposals), in fact, the most proposals were developed in a 2-level depth (16 proposals). Regarding, larger context models (more than 30 nodes) were presented in three depth levels 2, 4 and 5. On average, the proposed context models have 19.26 nodes and a depth level of 2.32 .

- Definition completeness: according to Fig. 5. we can observe that only $20 \%$ of the presented proposals have a unique and consistent definition for the context information specified in the context model (i.e. $100 \%$ definition completeness), either by explicitly defining the context in the paper or by referencing to the paper which has the definitions. Portion followed by $6 \%$ of well-defined context (i.e. between $80 \%$ and $90 \%$ definition completeness).

The remaining proposals present problems of different scale: (1) some context information is not defined; (2) the context model is based on several context models and although they are referenced, it is not specified which is the chosen definition for each context, leading to different definitions which are not consistent with each other; (3) the definitions on some context factors are too vague or ambiguous.

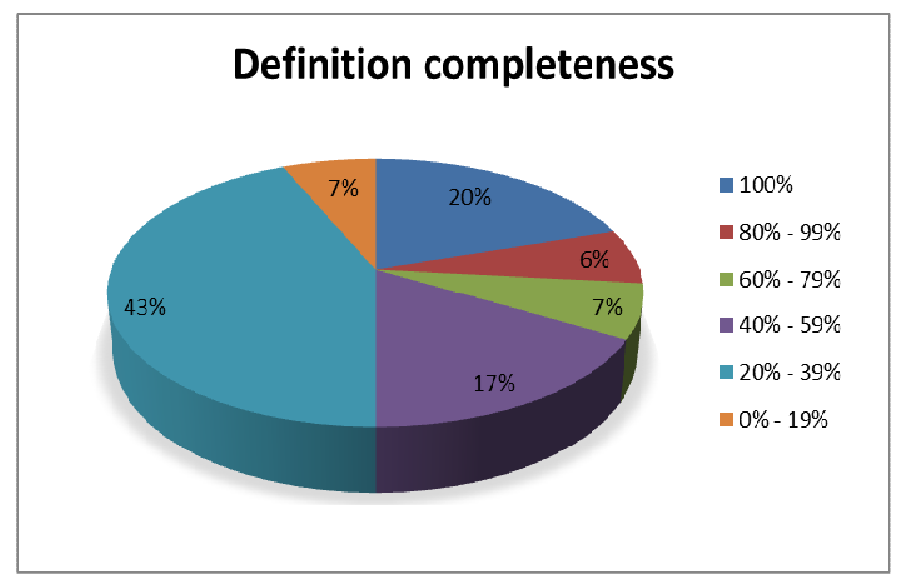

Fig. 5. Percentage of context models with their definition completeness.
Following the previous criteria, a majority of the presented proposals, up to $43 \%$ have a definition completeness between $20 \%$ and $39 \%$, i.e. between $20 \%$ and $39 \%$ of the contexts are defined within of the aforementioned problems. Another portion of $17 \%$ of context models has a definition completeness between $40 \%$ and $59 \%$, of context information defined with some of the aforementioned problems. $7 \%$ of the context models have a definition completeness between $60-79 \%$ and, the remaining $7 \%$ have a definition completeness between 0-19\%.

During the review of definition completeness we have performed a further analysis on each of the context definitions provided in the context model proposals. For brevity's sake, we focus only on the first hierarchy level of each context model proposed. In response, we observed two main issues, on the one hand a set of contexts are synonyms to each other, i.e., there are different context names referring to the same context meaning (e.g., location-place, environment-weather, etc.). On the other hand, a set of contexts are closely related to each other without being synonyms, i.e., they are subclasses of a higher context class, but this categorization is not clear. These aforementioned issues represent hierarchy definition problems, especially, because we are focusing on the same level hierarchy (e.g., physical and user environment clearly could be grouped in a single category called location). In this regard and in order to avoid inconsistencies as well as to unify context terms, we propose grouping synonyms and sub-classes into categories referring to a higher context concept as shown in TABLE III.

TABLE III. CONTEXT GROUPED.

\begin{tabular}{|c|c|c|c|}
\hline Category & Context grouped & Category & Context grouped \\
\hline Environment & $\begin{array}{l}\text { Physical } \\
\text { environment, User } \\
\text { environment, } \\
\text { Environmental } \\
\text { parameters, } \\
\text { Environmental, } \\
\text { Pollution }\end{array}$ & Preferences & $\begin{array}{l}\text { Individual } \\
\text { dimension, } \\
\text { Individuality, } \\
\text { Personal, Interest- } \\
\text { topic, interest, } \\
\text { Thing, Cognitive } \\
\text { pattern }\end{array}$ \\
\hline Location & $\begin{array}{lr}\text { Space, } & \text { Place, } \\
\text { Spatial, } & \text { Living } \\
\text { conditions, } & \text { Physical, } \\
\text { Indoor, Outdoor }\end{array}$ & Time & $\begin{array}{l}\text { Timestamp, } \\
\text { Physical, temporal, } \\
\text { sporadic, periodic }\end{array}$ \\
\hline Infrastructure & $\begin{array}{l}\text { Technical, } \\
\text { Technological, } \\
\text { Energy, Functional, } \\
\text { Non-functional, } \\
\text { Quality of service, } \\
\text { QoWS }\end{array}$ & Activity & $\begin{array}{l}\text { Attention, State, } \\
\text { Goals, Tasks, } \\
\text { Object, Schedule, } \\
\text { User history, } \\
\text { Action, Agenda } \\
\text { User action, } \\
\text { Event }\end{array}$ \\
\hline $\begin{array}{l}\text { Human } \\
\text { Factors }\end{array}$ & $\begin{array}{l}\text { Relations, Medical } \\
\text { Social dimension, } \\
\text { Relationship, Agent, } \\
\text { Community, contact, } \\
\text { Peers, Auxiliary, }\end{array}$ & Role & $\begin{array}{l}\text { Division } \\
\text { Labour, } \\
\text { Holder, } \\
\text { User role }\end{array}$ \\
\hline Policy & $\begin{array}{l}\text { Rules, } \\
\text { Legal entity }\end{array}$ & Entity & $\begin{array}{l}\text { User, Person, } \\
\text { Service, Provider, } \\
\text { third-party services }\end{array}$ \\
\hline Profiles & \multicolumn{3}{|c|}{$\begin{array}{l}\text { Information, characteristics, ability, education, profession, } \\
\text { expertise. Domain, Subscription information, identity }\end{array}$} \\
\hline Resource & \multicolumn{3}{|c|}{$\begin{array}{l}\text { Interaction means, Resource management, Resource } \\
\text { specification, Informational, Tools, Service, Economic, } \\
\text { Device, Platform, Network, Application, System, Computing, } \\
\text { physical object, sensor, interface, connectivity, } \\
\text { Computational, verbal, Value-added }\end{array}$} \\
\hline
\end{tabular}


It is important to mention that we are not making a formal reusing of the ontologies reviewed in the state of the art, since several proposals have specified the same context information at different context levels (hierarchies) and makes it difficult to reuse them. Therefore, we make a matching process among the context information proposed in which is only evaluated the first context level.

\section{CONCEPTUALIZATION}

As we have mentioned before, conceptualization phase structures the domain knowledge in a conceptual model that describes the problem and its solution in terms of the domain vocabulary identified in the ontology specification and acquisition knowledge. To carry out the conceptualization we use a glossary of terms and concept taxonomies to provide the conceptual model of the ontology corresponding to a high level hierarchy view. Hence, the first task to do is building a glossary of terms, for brevity's sake the glossary only includes concepts already identified in the stage of acquisition knowledge corresponding to the first hierarchy level of each context model proposed.

The glossary of terms depicted in TABLE IV identifies and gathers all the useful and potentially usable domain knowledge and its meanings. It is important to mention, that we have made an extended glossary of terms available in [67] (omitted here for space issues) providing definitions of every concept, property and entity presented in each of the analysed context models (if they were provided). Moreover, for issues related to inconsistent definitions, we tailor the definitions provided in the analysed context models, into definitions easy to understand in our domain (See TABLE IV).

TABLE IV.

GLOSSARY OF TERMS.

\begin{tabular}{|ll|}
\hline Main context & \multicolumn{1}{c|}{ Description } \\
\hline Time & $\begin{array}{l}\text { Time in a particular situation when an activity occurs, i.e., } \\
\text { time when an entity is involved on the interaction process } \\
\text { of service provisioning and consumption. }\end{array}$ \\
\hline Location & $\begin{array}{l}\text { Abstraction of a physical spatial place where an entity } \\
\text { interact with a service }\end{array}$ \\
\hline Environment & $\begin{array}{l}\text { Activities occurring near the entity and by the entity, } \\
\text { before, during or after the interaction with a service. }\end{array}$ \\
\hline Parameters of the environment such as light level, ambient \\
noise, temperature, humidity, etc., affecting the service \\
provisioning and consumption.
\end{tabular}

Once the glossary of terms contained a sizable number of terms, the next task is building concept taxonomies or conceptclassification trees, selecting terms that are concepts from the glossary of terms and structure their taxonomic relations. The resulting context taxonomy defining a conceptual model of the ontology corresponding to a high level hierarchy view is shown in Fig. 6.

In order to avoid possible errors in the upper ontology presented, it is important to examine and evaluate certain features of the ontology following the guidelines presented in [3][9]. The ontology evaluation mainly consist in verifying elements not simultaneously instance of two classes of a disjoint decomposition, checking that there are not loops in the concept taxonomy, verifying that several terms do not refer to the same concept and so on.

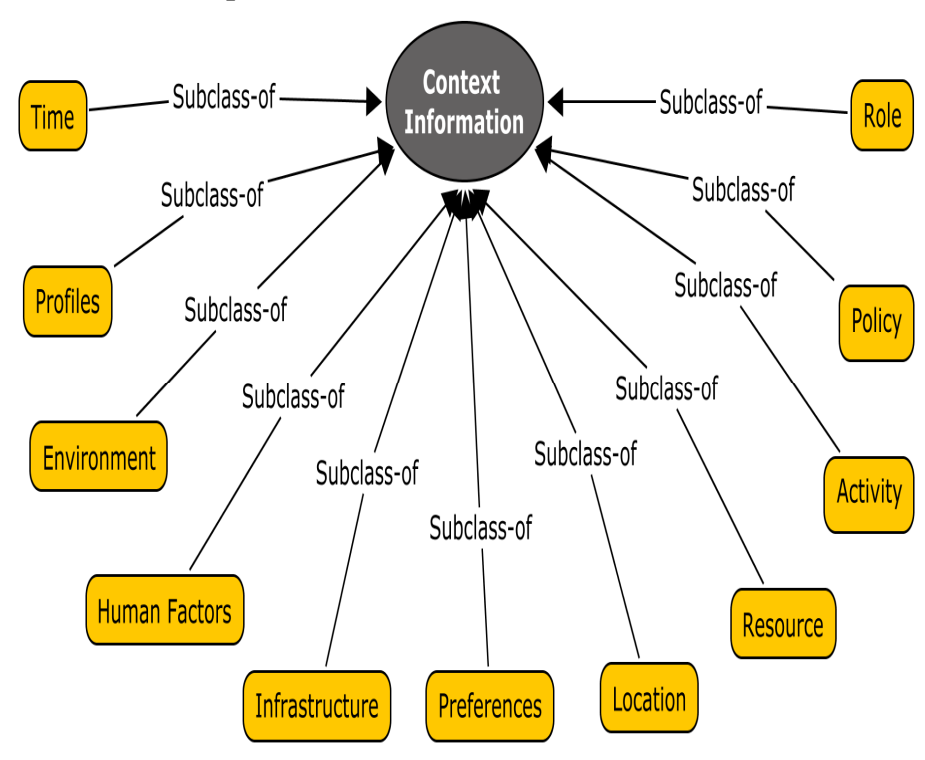

Fig. 6. Upper ontology proposed.

\section{ONTOLOGY INSTANTIATING, A SCENARIO}

In this section is addressed a scenario describing general concepts of the ontology through the relations among them. Normally to represent context information in different situations of the real word, a specialized scheme is created. In this regard, we propose an instantiation of the resulting ontology based on a demonstration scenario establishing a common context vocabulary including some properties and relations already identified in the activity of acquisition knowledge. The following scenario provides a high-level description usage scene of a Smart Parking service from the end-user's viewpoint.

The scenario describes in a nutshell a Smart Parking service allowing detecting available parking spots by placing the node under the pavement. It works with a magnetic sensor which detects when a vehicle is present or not. This monitoring platform is in charge to provide the information about each parking place to manage the available spaces. So, the usage scenario is as follows: 
Chris is a 50-year old business man that needs to attend an event in the centre of Barcelona and he is thinking to use his car to reach there, but he is worried for the availability of parking spots because it is a very busy place. One of his friends advise him to use a monitoring system available on the Web called Smart Parking Platform (SPP) to check free spaces in the area of the event and thus book a parking. SPP uses a sensor network that continuously monitors parking spot activity and incoming reservation requests, based on this information SPP website shows a map with lights indicators, green (unoccupied), yellow (reserved) and red (occupied).

Once Chris has booked parking through SPP website the system updates the map with lights indicators from green to yellow. Then, just before entering the garage, a system in place requires ID number of reservation request which was sent through SPP website to his smartphone. After validating the $I D$, the system updates the light indicator on the spot from yellow to red. Once the event has finished, Chris tries returning to the spot where his car is parked, but there is a difficulty, he does not remember the accurate spot, for this, Chris uses a SPP mobile application that from his current location and by means of Google Street view Chris is guided to the parking spot. Finally when Chris is gone, the system switched the light back to green and charged a parking fee to the driver's account.

As can be seen, the above scenario involves various context entities, concepts, relations and properties, which are depicted in the proposed instantiation illustrated in Fig. 7. through the associations that exist among one another.

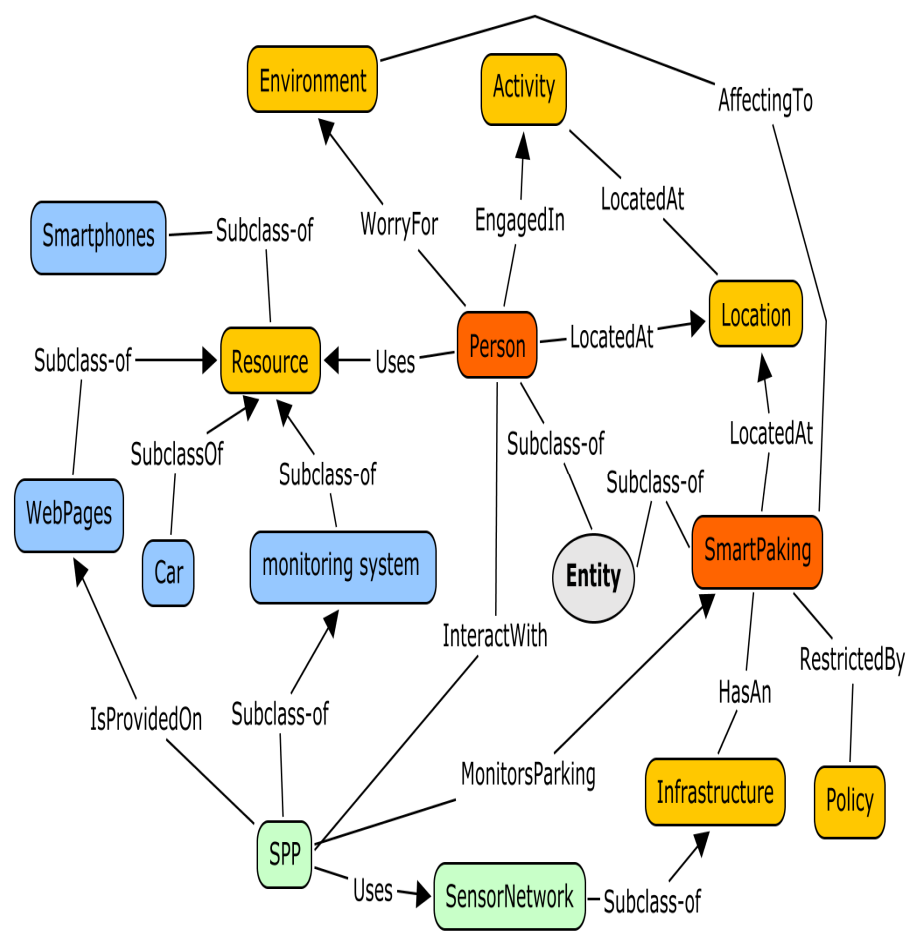

Fig. 7. Context ontology instantiation.

\section{CONCLUSIONS AND FUTURE WORK}

We have surveyed the state of the art of context model proposals for software services providing an overview on the field and making available a unified and consolidated upper ontology-based context, easy to be instantiated independent of the acting entity, specifically in the process of service provisioning and consumption from a service-centric perspective. Although we are focusing in a domain perspective we expect more generic applications and even allowing the integration and reuse of definitions already established into other ontologies as part of the essence imbued in our ontology.

As a result we provide a well-defined and consolidated infrastructure of context information defining a common body of knowledge that could be instantiated on variety of use cases such as monitoring and adaptation of different entities (e.g., services, users, providers, etc.), helping in service development process, or to be used as context information that allows knowing which contexts affect an entity before, during and after the service provisioning and consumption, among others. In this regard, a use case scenario has been proposed to demonstrate the instantiation options of the ontology in a real case.

As a final conclusion, we believe that this snapshot view on the anatomy of context models for software services may be a good reference for prospective researchers and practitioners on the field, especially with the aim of avoiding new classifications or definitions that may be contrary to the established practices that we have found. Regarding to the upper ontology provided, we believe that it can be used as a reference model, in order to alleviate different issues such as the provisioning and establishment of context hierarchies, inconsistencies on context factor definitions, among others. This work is intended to be adequately generic encompassing all the present and future particularities of context information seen from a service perspective.

As future work, we want to extend the state of the art addressed in this work in a form of systematic mapping considering an extended panoramic view of context model proposals. In addition, based on the context models retrieved and selected we want to improve the results provided in this paper. Also would be interesting to make a questionnaire to the entities identified in the service-centric perspective to provide percentages of which context surrounding them daily. One question could be as follows, what contexts provided in the context model are most related to your surrounding environment?

As final comment, this proposal is not intended to be completed since we only have considered the first level context information provided for each of the proposals analysed and evaluated. As future work we want to extend this perspective and consider the remaining context levels to complete our approach. Once this phase is reached, we are thinking to provide different resources from an ontological point of view, such as the instantiation method, inference, reasoning and implementation, etc. 


\section{ACKNOWLEDGMENT}

This work was partially supported by the Spanish project TIN2010-19130-C02-01. Oscar Cabrera is a PhD student at the UPC using a CONACYT grant.

\section{REFERENCES}

[1] J. Hong, E. Suh and S. Kim, "Context-aware systems: a literature review and classification," Expert Syst. Appl. 36, 8509-8522, 2009.

[2] J. Coutaz, J. L. Crowley, S. Dobson, and D. Garlan, "Context is key," Communications of the ACM, 48(3):49-53, 2005.

[3] A. Dey, "Providing architectural support for building context-aware Applications," PhD thesis, Atlanta, GA, USA, 2000.

[4] A. Dey, "Understanding and using context," Personal Ubiquitous Computing, 5(1):4-7, 2001.

[5] M. Fernández-López, A. Gómez-Pérez and N. Juristo, "METHONTOLOGY: from ontological art towards ontological engineering," Spring Symposium on Ontological Engineering of AAAI. Stanford University, California, pp 33-40, 1997.

[6] M. Fernández-López, A. Gómez-Pérez, A. Pazos and J. Pazos, "Building a chemical ontology using methontology and the ontology design environment," IEEE Intelligent Systems \& their applications 4(1):37-46, 1999.

[7] O. Corcho, M. López, A. Gómez-Pérez, and A. López-Cima, "Building legal ontologies with METHONTOLOGY and WebODE," in Lecture Notes in Computer Science - Law and the Semantic Web, Ed: E. S. B. H. I.-P.-. PP. 142-157, 2005.

[8] Office of Government Commerce (OGC), "ITIL Version 3 - Service strategy," United Kingdom, 2007.

[9] C. Grönroos, "Service logic revisited: who creates value? and who cocreates?," European Business Review, 20(4), 298-314, 2008.

[10] OASIS, "Reference model for service oriented architecture," 2006.

[11] M. Papazoglou, "Service-oriented computing: concepts, characteristics and directions," In Web Information Systems Engineering. WISE. Proceedings of the Fourth International Conference. IEEE, 2013.

[12] "Context." Merriam-Webster.com. Accessed February 15, 2014. http://www.merriam-webster.com/dictionary/context.

[13] P. Korpipaa, J. Mantyjarvi, J. Kela, H. Keranen and E. Malm, "Managing context information in mobile devices," IEEE Pervasive Computing 2 (3), 42-51, 2003.

[14] O. Kwon, "Modeling and generating context-aware agent-based applications with amended colored petri nets," Expert Systems with Applications 27 (4), 609 - 621, 2004.

[15] E. Badidi and L. Esmahi, "A cloud-based approach for context information provisioning," arXiv preprint arXiv:1105.2213, 2011.

[16] P. Brown, "The Stick-e document: a framework for creating contextaware applications," In the Proceedings of the Electronic Publishing, pp. 259-272, Laxenburg, Austria, IFIP, 1996.

[17] T. Buchholz, A. Kpper and M. Schiffers, "Quality of context: what it is and why we need it?," In Proc. of the 10th International Workshop of the HP OpenView University association (HPOVUA), 2003.

[18] T. Gu, H. Pung, and D. Zhang, "A service-oriented middleware for building context-aware services," J. Netw. Comput. Appl. 28, 1, 1-18, 2005.

[19] H. Byun and K. Cheverst, "Utilizing context history to provide dynamic adaptations," Applied Artificial Intelligence 18 (6), 533-548, 2004.

[20] A. Schmidt, "Context-aware computing: context-awareness, contextaware user interfaces, and implicit interaction," The Encyclopedia of Human-Computer Interaction, 2nd Ed, 2013.

[21] J. Crowley, J. Coutaz, G. Rey and P. Reignier, "Perceptual components for context-aware computing," In Proceedings of the Fourth International Conference of Ubiquitous Computing, (Göteburg, Sweden). Springer, 117-134, 2002.

[22] N. Noy and D. McGuiness, "Ontology development 101: a guide to creating your first ontology," Stanford Knowledge Systems Laboratory Technical Report KSL-01-05, 2001.
[23] N. Noy, "Semantic integration: a survey of ontology-based approaches," SIGMOD Record, 33(4), 2004.

[24] H. Chen, F. Perich, T. Finin, and A. Joshi, "SOUPA: standard ontology for ubiquitous and pervasive applications," In 1st Annual Int'1 Conf. on Mobile and Ubiquitous Systems: Networking and Services, 2004.

[25] X. Wang, D. Zhang, T. Gu, and H. Pung "Ontology based context modeling and reasoning using OWL," In Pervasive Computing and Communications Workshops, 2004. Proceedings of the Second IEEE Annual Conference on (pp. 18-22). IEEE, 2004.

[26] K. Sudhana, V. Raj, and R. Suresh, "An ontology-based framework for context-aware adaptive e-learning system," In Computer Communication and Informatics (ICCCI), International Conference on (pp. 1-6). IEEE, 2013.

[27] M. Uschold and M. King, "Towards a methodology for building ontologies," In: Skuce D (eds) IJCAI'95 Workshop on Basic Ontological Issues in Knowledge Sharing. Montreal, Canada, pp 6.16.10, 1995.

[28] M. Gruninger and M. Fox, "Methodology for the design and evaluation of ontologies," In: Proceedings of the Workshop on Basic Ontological Issues in Knowledge Sharing, IJCAI-95, Montreal, 1995.

[29] S. Staab, HP. Schnurr, R. Studer and Y. Sure, "Knowledge processes and ontologies," IEEE Intelligent Systems 16(1):26-34, 2001.

[30] M. Uschold and M. Gruninger, "Ontologies principles, methods and applications knowledge engineering review," Vol. 11; N. 2, 1996.

[31] A. Gómez-Pérez, "Evaluation of ontologies," International Journal of Intelligent Systems 16(3):391-409, 2001.

[32] M. Uschold, "Building ontologies: towards a unified methodology," Technical report-university of Edinburgh AI applications institute AIAI tr, 1996.

[33] C. Bolchini, C. Curino, E. Quintarelli, F. Schreiber, and L. Tanca, "A data-oriented survey of context models," SIGMOD Rec., 36:19-26, 2007.

[34] W. Schilit, N. Adams, and R. Want, "Context-aware Computing Applications," In the Proceedings of the 1st International Workshop on Mobile Computing Systems and Applications, pp. 85-90, Santa Cruz, CA, IEEE, 1994.

[35] G. Chen and D. Kotz, "A survey of context-aware mobile computing research," Technical report, Hanover, NH, USA, 2000.

[36] K. Mitchell, "Supporting the development of mobile context-aware computing," PhD thesis, Lancaster University, 2002.

[37] A. Schmidt, M. Beigl and H. W. Gellersen, "There is more to context than location," Computers \& Graphics, 23(6), 893-901, 1999.

[38] D. Preuveneers, J. Van den Bergh, D. Wagelaar, A. Georges, P. Rigole, T. Clerckx, Y. Berbers, K. Connix and V. Jonckers, "Towards an extensible context ontology for ambient intelligence," 2004.

[39] H. Kranenburg, M. Bargh, S. Iacob, and A. Peddemors, "A context management framework for supporting context-aware distributed applications," IEEE Communications Magazine, 44, 8, 67-74, 2006.

[40] A. Zimmermann, A. Lorenz, and R. Oppermann, "An operational definition of context," In Proceedings of the 6th International and Interdisciplinary Conference on Modeling and using Context (CONTEXT07), 558-571, 2007.

[41] B. Medjahed and Y. Atif, "Context-based matching for Web service composition," Distributed and Parallel Databases, 21(1), 5-37, 2007.

[42] P. Eugster, B. Garbinato, and A. Holzer, "Middleware support for context-aware applications," In Middleware for Network Eccentric and Mobile Applications, B. Garbinato, H.Miranda, and L. Rodrigues, Eds. Springer Press, Berlin, Chapter 14, 305-322, 2009.

[43] K. Bandara, M.Wang, and C. Pahl, "Context modeling and constraints binding in Web service business processes," In Proceedings of the first international workshop on Context-aware software technology and applications, CASTA'09, pages 29-32, New York, NY, USA, ACM, 2009.

[44] T. Stavropoulos, D. Vrakas, D. Vlachava, and N. Bassiliades, "BOnSAI: a Smart Building Ontology for Ambient Intelligence," in WIMS'12 Proceedings of the 2nd International Conference on Web Intelligence, Mining and Semantics, 2012. 
[45] A. Ranganathan, R. E. McGrath, R. H. Campbell and M. D. Mickunas, "Ontologies in a pervasive computing environment," In Workshop on Ontologies in Distributed Systems, IJCAI, Acapulco, México, 2003.

[46] M. Grassi, M. Nucci, and F. Piazza, "Ontologies for smart homes and energy management: an implementation-driven survey," In Modeling and Simulation of Cyber-Physical Energy Systems (MSCPES), Workshop on, pp. 1-3. IEEE, 2013.

[47] C. Niederée, A. Stewart, B. Mehta, and M. Hemmje, "A multidimensional, unified user model for cross-system personalization," In E4PIA Workshop, 2004.

[48] M. Kaenampornpan and E. O'Neill, “An integrated context model: bringing activity to context," In Proc. Workshop on Advanced Context Modelling, Reasoning and Management, 2004.

[49] M. Strimpakou, I. Roussaki and M. Anagnostou, "A context ontology for pervasive service provision," In 20th Int. Conf. on Advanced Information Networking and Applications, pages 775-779, 2006.

[50] C. Bolchini, C. Curino, E. Quintarelli, F. A. Schreiber and L. Tanca, "Context-ADDICT," Technical Report 2006.044, Dip. Elettronica e Informazione, Politecnico di Milano, 2006.

[51] K. Henricksen, J. Indulska1 and A. Rakotonirainy, "Modeling context information in pervasive computing systems," In Proc. 1st Intl Conf. on Pervasive Computing, LNCS 2414, pages 167-180, 2002.

[52] H. Yang, A. Huang, R. Chen, S.-S. Tseng and Y.-S. Shen, "Context model and context acquisition for ubiquitous content access in ulearning environments," In IEEE Int. Conf. Sensor Networks, Ubiquitous, and Trustworthy Computing, volume 2, pages 78-83, 2006.

[53] M. Blake, D. Kahan, and M. Nowlan, "Context-aware agents for useroriented Web services discovery and execution," Distrib. Parallel Databases, 21(1):39-58, February 2007.

[54] M. Golemati, A. Katifori, C. Vassilakis, G. Lepouras and C. Halatsis, "Creating an ontology for the user profile: method and applications," In Proc. RCIS, pages 407-412, 2007.

[55] H. Shen and Y. Cheng, "A semantic context-based model for mobile Web services access control," volume 3, pages 18-25. 2011.

[56] K. Pathan, S. Reiff-Marganiec, A. Shaikh and N. Channa, "Reaching activities by places in the context-aware environments using software sensors," Journal of Emerging Trends in Computing and Information Sciences, 2:665-673, 2011.
[57] S. Scerri, J. Attard, I. Rivera, M. Valla and S. Handschuh, "Dcon: interoperable context representation for pervasive environments," In Proc. of the Activity Context Representation Workshop at AAAI, 2012.

[58] J. Kim and K. Chung, "Ontology-based healthcare context information model to implement ubiquitous environment," Multimedia Tools and Applications, pages 1-16, 2013.

[59] A. Dix, T. Rodden, N. Davies, J. Trevor, A. Friday and P. Palfreyman, "Exploiting Space and Location as a Design Framework for Interactive Mobile Systems," ACM Transactions on Computer-Human Interaction (TOCHI). 1999.

[60] D. Brickley and L. Miller, "FOAF vocabulary specification," In RDFWeb Namespace Document. RDFWeb, xmlns.com, 2003.

[61] F. Pan and J. R. Hobbs, "Time in OWL-S," In Proceedings of AAAI-04 Spring Symposium on SemanticWeb Services, Stanford University, California, 2004.

[62] D. Lenat and R. Guha, "Building large knowledge-based systems: representation and inference in the CycProject," Addison-Wesley, 1990.

[63] D. Randell, Z. Cui and A. Cohn, "A spatial logic based on regions and connection," In B. Nebel, C. Rich, and W. Swartout, editors, KR'92. Principles of Knowledge Representation and Reasoning: Proceedings of the Third International Conference, pages 165-176. Morgan Kaufmann, San Mateo, California, 1992.

[64] F. Perich, "MoGATU BDI Ontology," 2004.

[65] L. Kagal, T. Finin and A. Joshi, "A policy based approach to security for the semantic Web," In 2nd International Semantic Web Conference (ISWC2003), 2003.

[66] H. Chen, T. Finin and A. Joshi, "An ontology for context-aware pervasive computing environments,” Knowl. Eng. Rev., 18(3):197-207, 2003.

[67] O. Cabrera, X. Franch and J. Marco, "Appendix of: a context ontology for service provisioning and consumption," 2014. [Online]. Available: http://gessi.lsi.upc.edu/qcontextmodels/. 